\title{
First observation of Edge Localized Modes mitigation with resonant and non-resonant magnetic perturbations in ASDEX Upgrade
}

\author{
W. Suttrop, T. Eich, J. C. Fuchs, S. Günter, A. Janzer, A. Herrmann, A. Kallenbach, \\ P. T. Lang, T. Lunt, M. Maraschek, R. M. McDermott, A. Mlynek, T. Pütterich, M. Rott, \\ T. Vierle, E. Wolfrum, Q. Yu, I. Zammuto, H. Zohm, and the ASDEX Upgrade Team \\ Max-Planck-Institut für Plasmaphysik, \\ Assoziation IPP-EURATOM, D-85748 Garching
}

(Dated: June 3, 2011)

\begin{abstract}
First experiments with non-axisymmetric magnetic perturbations, toroidal mode number $n=2$, produced by newly installed in-vessel saddle coils in the ASDEX Upgrade tokamak show significant reduction of plasma energy loss and peak divertor power load associated with type-I Edge Localized Modes (ELMs) in high-confinement mode plasmas. ELM mitigation is observed above an edge density threshold and is obtained both with magnetic perturbations that are resonant and not resonant with the edge safety factor profile. Compared with unperturbed type-I ELMy reference plasmas, plasmas with mitigated ELMs show similar confinement, similar plasma density and lower tungsten impurity concentration.
\end{abstract}

PACS numbers: 28.52.s, 52.55.Fa, 52.55.Rk 
For a tokamak fusion reactor, the mitigation of Edge Localized Modes (ELMs) is likely a mandatory requirement to avoid excessive erosion of the divertor targets and yet exploit the benefits of high confinement mode (H-mode) operation [1,2]. Application of non-axisymmetric error fields has early been found to affect ELMs in COMPASS-D [3]. In JET [4], a reduction of ELM losses was observed when applying perturbations with external coils normally used for the correction of field errors. Full suppression of ELMs has been achieved with in-vessel coils in DIII-D $[5,6]$ at low collisionality (measured at the $\mathrm{H}$-mode edge pedestal) with resonant perturbations and ELM mitigation at high collisionality with nonresonant perturbations.

ASDEX Upgrade [7] is presently being enhanced with a set of in-vessel saddle coils [8-10]. Four coils above (dubbed Bu coils) and four coils below the midplane (Bl coils) at the low field side (Fig. 1) are currently operational. These coils have five turns each and create a mainly radial field with toroidal mode numbers up to $n=2$. Another eight coils (allowing $n \leq 4$ ) are scheduled for installation during the next maintenance break. The toroidal coil arrangement is shown in Fig. 2. For the present experiments, $n=2$ perturbations are applied with either even or odd parity of the upper and lower coil currents as indicated in Fig. 2. Positive coil current corresponds to radially outward directed perturbation field. All B-coils are connected in series and are supplied with a single dc power converter.

The present experiments are conducted with plasma current $I_{p}=0.8$ or $I_{p}=1.0 \mathrm{MA}$, toroidal field between $B_{t}=2.3$ and $B_{t}=2.7 \mathrm{~T}$, corresponding to edge safety factors in the range of $q_{95}=4.8$ to 6.2. All plasmas are in lower single null divertor configuration (grad- $B$ drift towards $\mathrm{X}$-point) with low triangularity cross sections as shown in Fig. 1 which also outlines the geometry of the main diagnostics used. Time traces of discharge $26081, B_{t}=2.5 \mathrm{~T}$, are shown in Fig. 3. After plasma current ramp-up, an ELMing H-mode discharge is set up with neutral beams injected in codirection with the plasma current (power $P_{\mathrm{NBI}}=7.5 \mathrm{MW}$ ), second harmonic central electron cyclotron heating $\left(f=140 \mathrm{GHz}, P_{\mathrm{ECRH}}=1.4 \mathrm{MW}\right)$ and a gas puff of $9 \times 10^{21} \mathrm{D}$ atoms $/ \mathrm{s}$. The ELMs show all characteristics of type-I ELMs [11], in particular the positive heating power dependence of the ELM frequency $\left(f_{\mathrm{ELM}} \approx 50-75 \mathrm{~Hz}\right.$ for $\left.P_{\text {heat }}=5-7.5 \mathrm{MW}\right)$. After application of the $B$ coils, (odd parity, current $I_{\text {coil }}=0.9 \mathrm{kA} \cong 4.5 \mathrm{kAt}$, at $t=2 \mathrm{~s}$ ), the type-I ELMs become gradually less frequent (time interval marked " 1 " in Fig. 3) and eventually, after $t=2.8 \mathrm{~s}$, disappear entirely and give way to smaller transport events with high repetition frequency, $f \approx 400 \mathrm{~Hz}$ (time interval marked "2"). Since these events show many features of ELMs but with significantly reduced levels of energy and particle losses (see below), they are subsequently termed "mitigated ELMs" 
and phases where they replace large type-I ELMs "ELM-mitigated phases". They are characterized by reduced excursions on the peripheral line density [deuterated cyanide (DCN) interferometer chord], divertor D-alpha intensity, and divertor temperature, as derived from thermocurrents through divertor plates [12]. ASDEX Upgrade plasma facing components have tungsten surfaces and the relative core plasma tungsten concentration is routinely monitored by vacuum ultraviolet spectroscopy. During the ELM-mitigated phase, the core tungsten concentration obtained from line radiation (bottom trace) as described in Ref. [13] decreases by about a factor of 2 compared to the type-I ELMy H-mode flat top and remains below $10^{-5}$ of the plasma density during the $B$ coil phase. Tungsten accumulation is not observed.

A side-by-side comparison of characteristics of unmitigated and ELM-mitigated phases is shown in Fig. 4 and Fig. 5, respectively, for a discharge with identical waveforms as before, except the saddle coils are temporarily switched off during the H-mode flattop for $t=3.1-4.0 \mathrm{~s}$. With coils on, the ELM energy loss (measured by the MHD stored energy) is reduced from $60 \mathrm{~kJ}$ to less than $10 \mathrm{~kJ}$. The electron line density (peripheral chord as indicated in Fig. 1) remains near its peak values in the type-I ELMy phase. The pedestal top electron temperature reaches $T_{e}=520 \mathrm{eV}$ prior to type-I ELMs while it remains somewhat below, $T_{e} \leq 500 \mathrm{eV}$, in the ELM-mitigated phase. ELM mitigation is reflected by reduced excursions of all quantities plotted. The total power load is measured by infrared thermography. In unmitigated phases, the peak power caused by type-I ELMs is up to $8 \mathrm{MW}$ in the inner and up to $5 \mathrm{MW}$ in the outer divertor. In ELM-mitigated phases, the inner divertor remains detached at all times (divertor power less than $1 \mathrm{MW}$ ) and the outer divertor is exposed to a peak power load below 2.5 MW. The event-like nature of energy losses during the ELM-mitigated phase is demonstrated in the inset of Fig. 5. Small pedestal temperature crashes are followed by outer divertor target power peaks.

The necessary conditions to access the ELM mitigation scenario have not yet been fully explored. An apparent feature seems to be the existence of a critical minimum edge density. ELM mitigation is only observed above a peripheral line density of $\bar{n}_{\mathrm{e}, \text { edge }}=4.8 \times 10^{19} \mathrm{~m}^{-2}$ for $I_{p}=$ $0.8 \mathrm{MA}$ and $\bar{n}_{\mathrm{e}, \text { edge }}=6.5 \times 10^{19} \mathrm{~m}^{-2}$ for $I_{p}=1.0 \mathrm{MA}$. The neoclassical pedestal electron collisionality (defined as in Ref. [2]), determined from the ECE and interferometer measurements at the transition to ELM mitigation, corresponds to $v_{\mathrm{e}, \text { neo }}^{*} \approx 1.5\left(I_{p}=0.8 \mathrm{MA}\right)$ and $v_{\mathrm{e}, \text { neo }}^{*} \approx 3$ $\left(I_{p}=1.0 \mathrm{MA}\right)$. We note that the edge density in both cases is approximately at the same fraction $f_{G W}$ of the Greenwald density limit [14], namely $f_{G W}=0.63\left(I_{p}=0.8 \mathrm{MA}\right)$ and $f_{G W}=0.65$ $\left(I_{p}=1.0 \mathrm{MA}\right)$. Fig. 3 illustrates the transition to the ELM mitigation phase until $t=2.8 \mathrm{~s}$ (time 


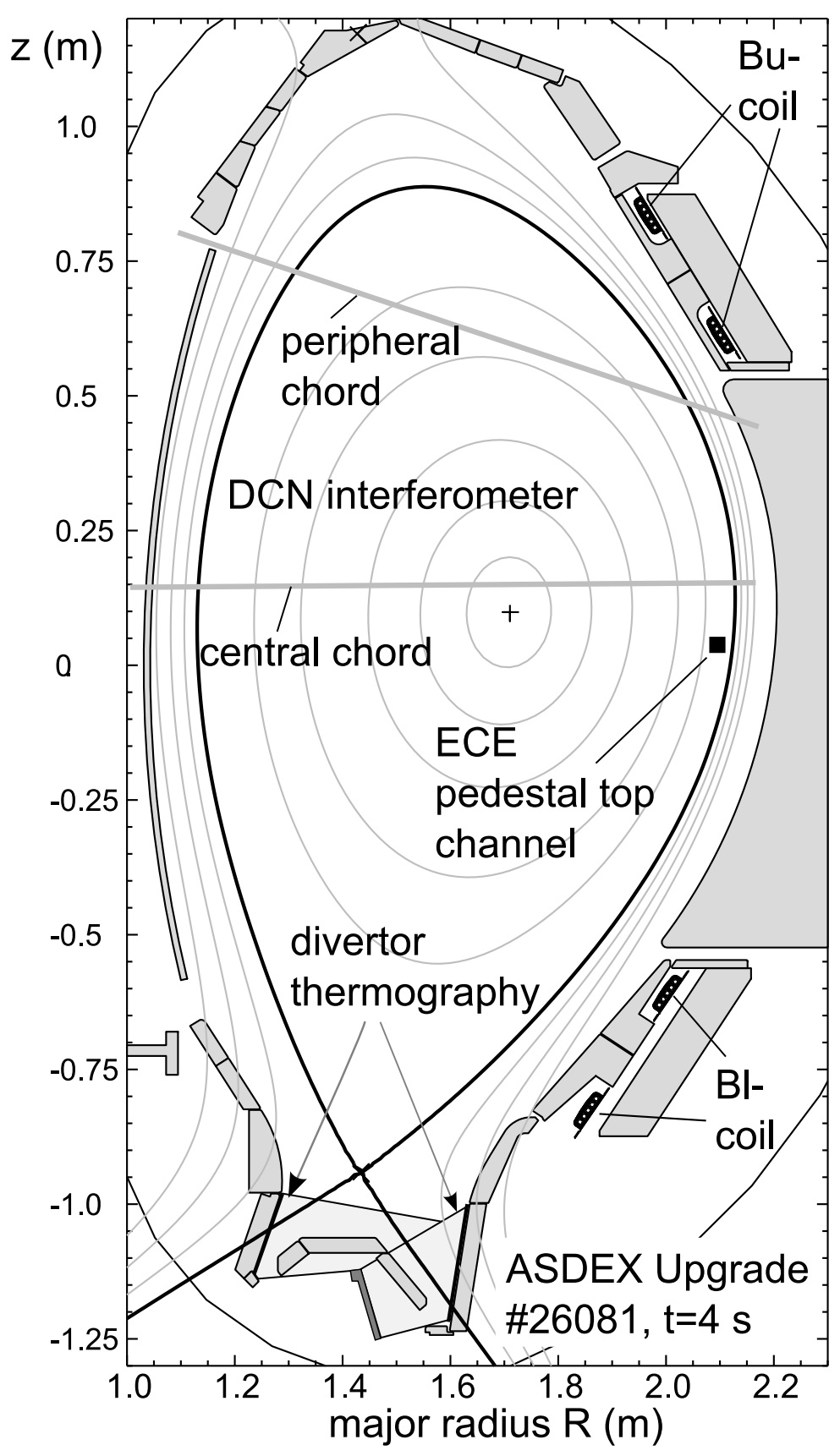

FIG. 1. Poloidal cross section of ASDEX Upgrade showing the locations of the $\mathrm{Bu}$ - and Bl-coils, the plasma cross section for the present experiments, interferometer chords, the location of the pedestal top ECE measurement and divertor thermography views.

interval 1). As the edge density approaches the threshold from below, the type-I ELM frequency is reduced and the type-I ELMs become interspersed with the small ELM-like transport events. Above the critical density, type-I ELMs disappear completely. There is no continuous evolution of ELM energy loss or peak divertor power between large and small ELM events. At the same gas 


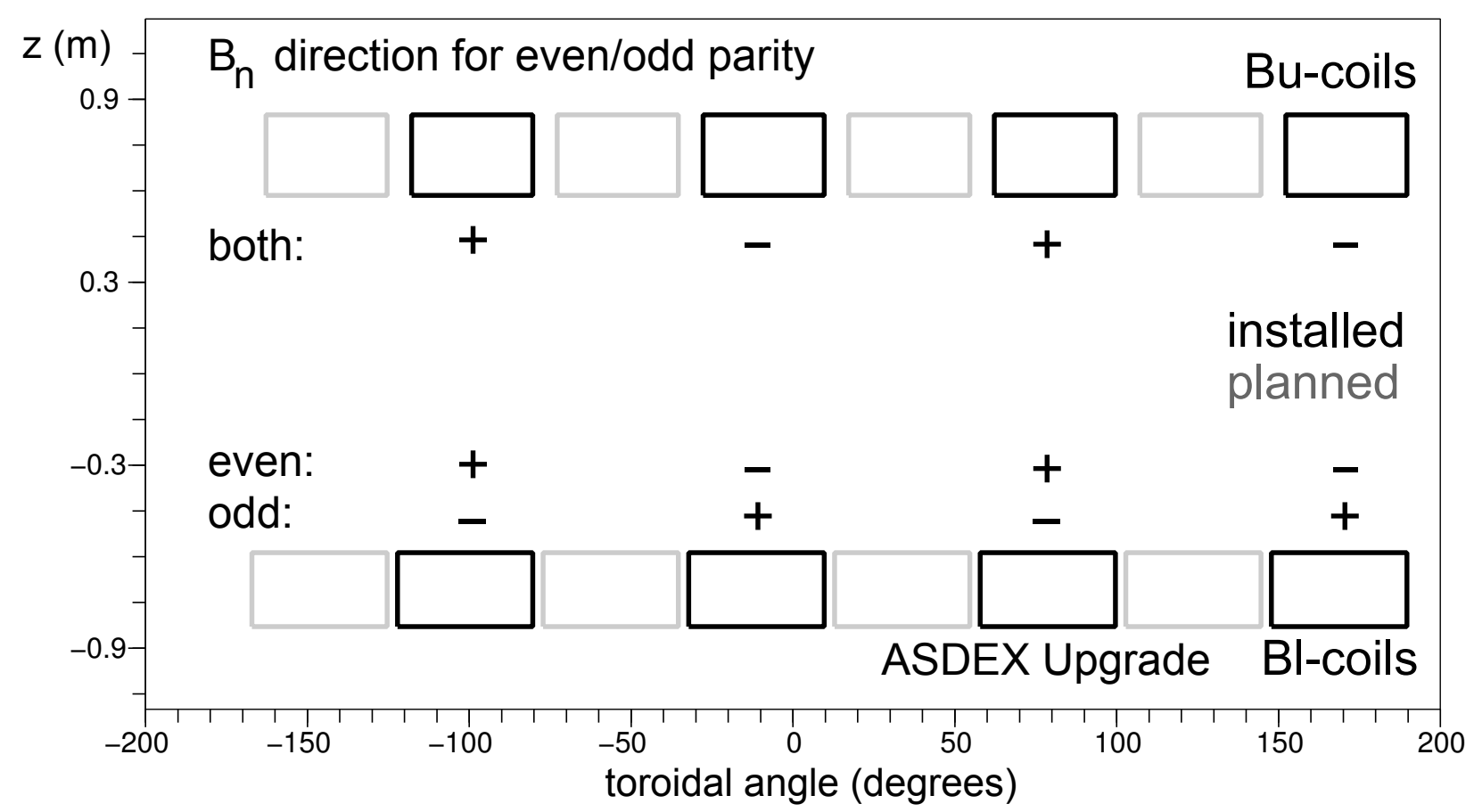

FIG. 2. Toroidal arrangement of the B-coils in operation (black) and those to be yet installed (gray); normal field direction in even and odd parity configurations.

fueling rate, the density in the mitigated phase is similar to or higher than in the preceding type-I ELMy phase; a "density pumpout" when applying the field perturbation is not observed in these discharges.

Toroidal plasma fluid rotation is measured by charge exchange recombination spectroscopy of the boron impurity $\mathrm{B}^{4+} n=7 \rightarrow 6$ transition (wavelength $494.467 \mathrm{~nm}$ ) using a heating neutral beam. The largest variation of neutral beam geometry and mix of NBI and ECRH power has so far been produced at a plasma current of $I_{p}=0.8 \mathrm{MA}$. For both type-I ELMy and ELM mitigated $\mathrm{H}$-mode plasmas the pedestal top toroidal rotation velocity ranges between 30 and $40 \mathrm{~km} / \mathrm{s}$. Within this range there is no apparent variation of the density threshold for ELM mitigation.

The required B-coil current for ELM mitigation is measured in two discharges with odd (26196) and even parity (26201) and otherwise similar parameters: $B_{t}=2.4 \mathrm{~T}, I_{p}=0.8 \mathrm{MA}$, $P_{\mathrm{NBI}}=7.5 \mathrm{MW}$ and $\bar{n}_{\mathrm{e}, \text { edge }}=5.7 \times 10^{19} \mathrm{~m}^{-2}$ at the time of the transition. Time traces are shown in Fig. 6. Figure 7 shows the poloidal mode number $m$ spectrum (perturbation field amplitude at the $q=5$ surface, at the pedestal top) for the principal toroidal mode number $n=2$. The $m=q \times n=10$ resonant amplitude is higher for the odd parity case by a factor of 5.5 compared to the even parity case. However, in both cases the last type-I ELM occurs at a coil current of 


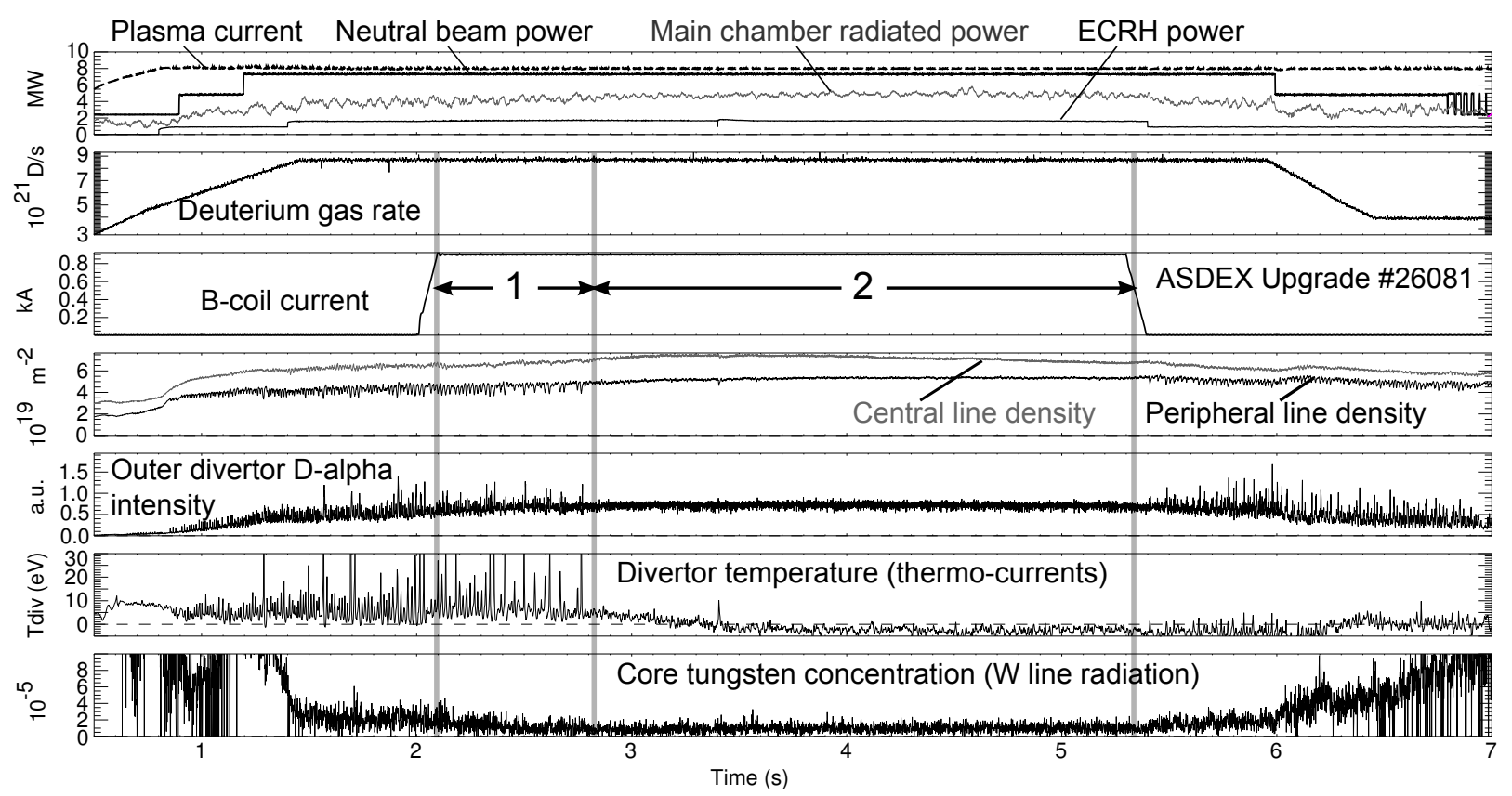

FIG. 3. Time traces of discharge 26081 with B-coils operating in odd parity (resonant configuration). Time intervals marked "1" and "2" show a reduction of type-I ELM frequency and full suppression of type-I ELMs, respectively.

$I_{\text {coil }}=350$ A; i.e. no threshold difference between optimum resonant and optimum nonresonant conditions is observed. The ELM-mitigated phase persists into the coil current ramp-down. At $I_{\text {coil }} \approx 150 \mathrm{~A}$, the edge density jumps up and the character of the mitigated ELMs changes towards larger D-alpha excursions. Large type-I ELMs re-occur only after the coils are completely switched off. The reasons for this apparent hysteresis are unknown as yet. The safety factor has been varied between $q_{95}=4.8$ and $q_{95}=6.2$ by means of slow $B_{t}$ ramps at fixed $I_{p}=0.8 \mathrm{MA}$. With B-coils operated in odd parity $\left(I_{\text {coil }}=900 \mathrm{~A}\right)$ ELM mitigation is observed in the entire $q_{95}$ range covered. With even parity, a range of $q_{95}=5.0-5.8$ has been scanned, and type-I ELMs re-appear at $q_{95}<5.3$, neither at maximum nor at minimum resonant field.

In summary, the first experiments with non-axisymmetric magnetic $n=2$ perturbations produced by newly installed in-vessel saddle coils in ASDEX Upgrade demonstrate clear mitigation of ELMs in H-mode plasmas. In the plasmas studied so far, type-I ELMs are replaced by a distinctly different type of ELM-like transport event which is typically characterized by a reduction of divertor power by a factor of 8 in the inner and 2 in the outer divertor and a reduction of core plasma energy loss by a factor of 6 . As yet, no performance penalty of the ELM-mitigated regime has been encountered. Stored energy, and pedestal top density remain at the pre-type-I ELM val- 
ues. The pedestal electron temperature is reduced by an amount much smaller than the temperature excursions due to type-I ELMs. The residual core tungsten concentration is at the same level or lower than in comparable type-I ELMy phases. Operation of the coils with a $n=2$ configuration has not led to plasma termination, neither by tungsten accumulation nor by locking of tearing modes.

Among the ELM mitigation scenarios encountered in other tokamaks, this ELM mitigation regime, obtained with pedestal collisionalities $v_{\mathrm{e}, \text { neo }}^{*} \geq 1.5\left(I_{p}=0.8 \mathrm{MA}\right)$, compares best with the
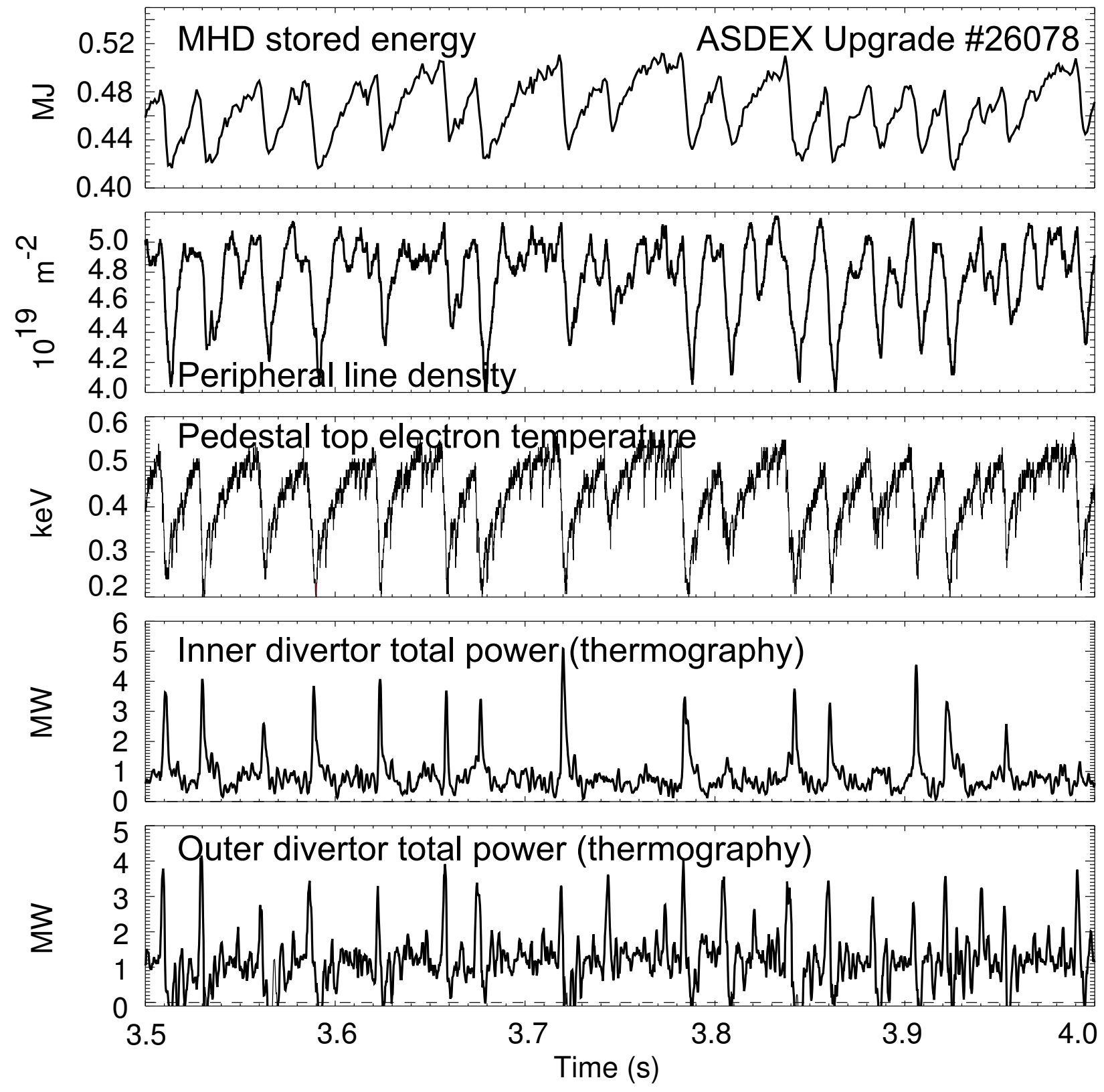

FIG. 4. Time traces for an unmitigated ELMy phase (B-coils off). 
high-collisionality regime of DIII-D [6]. However, the ASDEX Upgrade data does not support a collisionality threshold. Also, while in DIII-D ELM mitigation at high collisionality occurs only in a narrow window of $\Delta q_{95} \pm 0.2$ around $q_{95}=3.7$ [6], there seems to be a wide safety factor access range in ASDEX Upgrade. In particular, setting $q_{95}$ and coil current parity to optimum resonant or non-resonant conditions, as identified by calculating mode spectra on the basis of numerically reconstructed plasma equilibria, does not lead to the observation of different access conditions

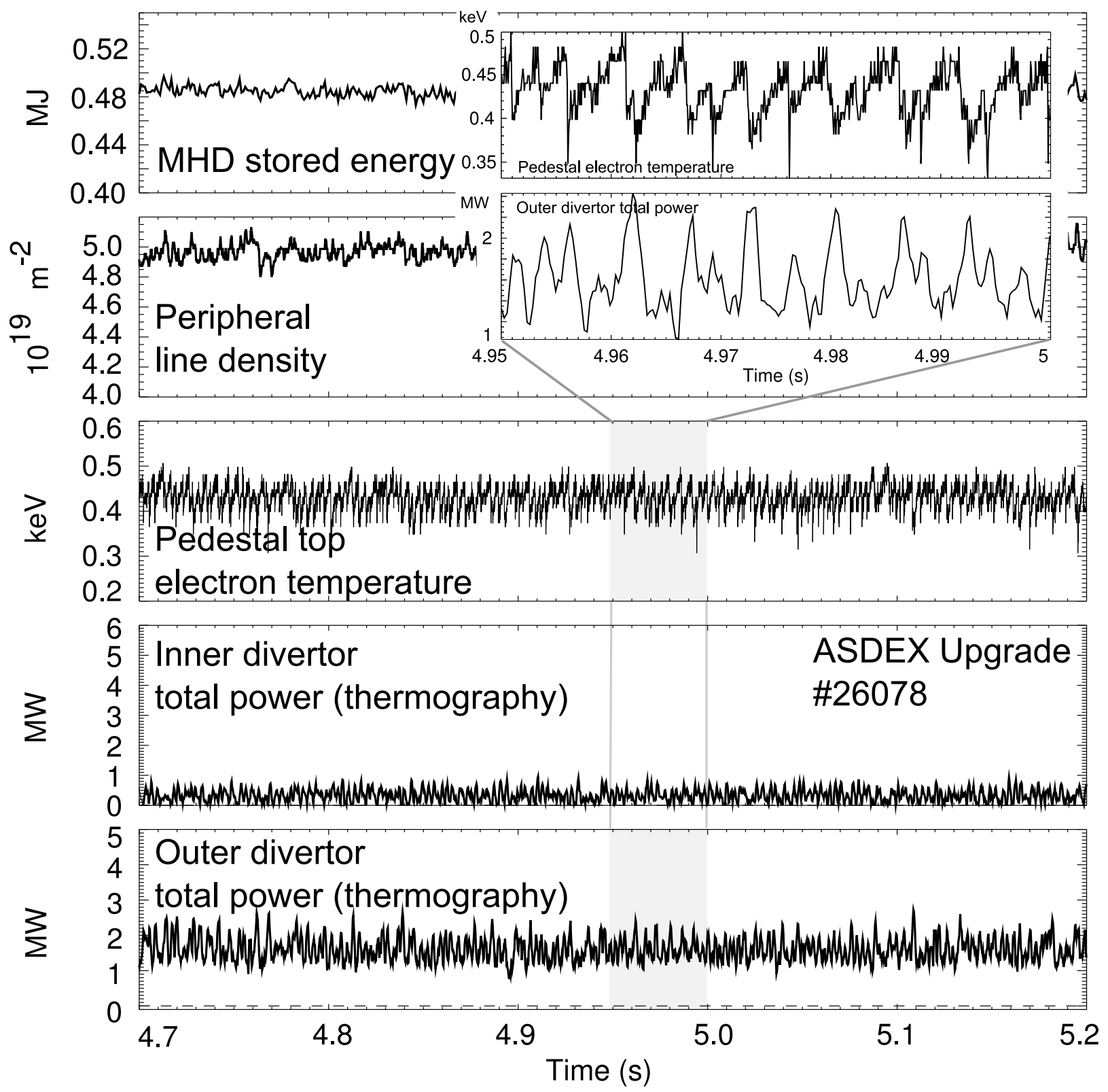

FIG. 5. Time traces for a phase with mitigated ELMs (B-coils operating with odd parity). The insert shows small transport events as seen in pedestal top electron temperature and outer divertor power. 

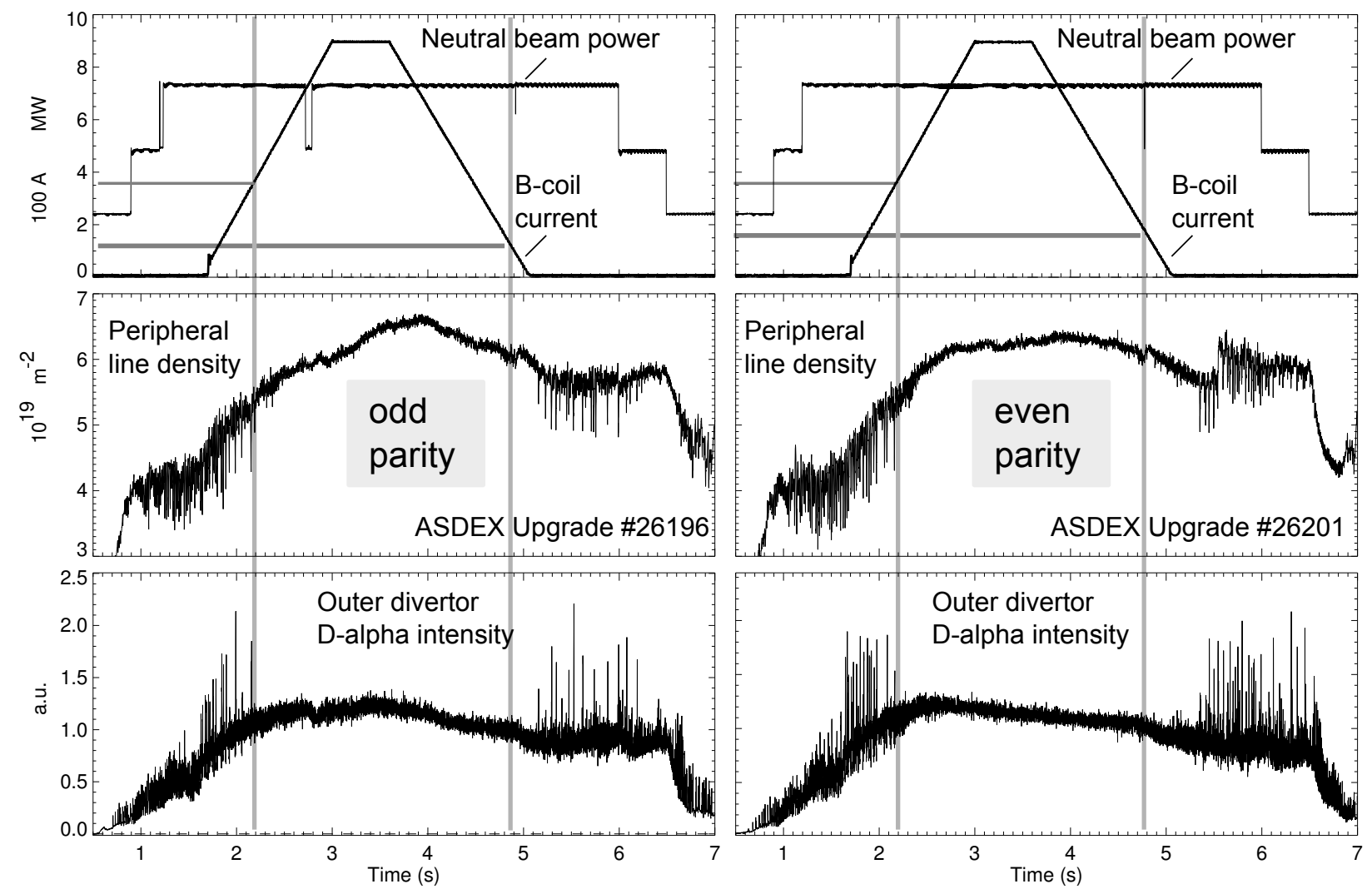

FIG. 6. Measurement of coil current threshold for ELM mitigation with odd (left) and even (right) parity.

or any other distinct experimental feature. It should be noted that in all cases so far, application of the $n=2$ perturbation has not led to observable effects (acceleration or deceleration) on the toroidal rotation velocity in the plasma interior. This and the apparent absence of locked magnetic islands in the H-mode edge might be indications of weak field penetration into the pedestal and core regions. The apparent lack of sensitivity of the ELM mitigation effect to resonances on closed flux surfaces, especially the sharp nonresonant notches, opens up questions about the underlying physics of ELM mitigation. The smaller transport events are triggered at consistently smaller pedestal temperature than encountered just before type-I ELMs. This might lead to the speculation that the magnetic perturbation introduces a lower stability boundary for small ELMs, which is encountered before large type-I ELMs are triggered.

Further studies in ASDEX Upgrade will aim at exploring the regime boundaries towards lower $q_{95}$ and lower edge collisionality and improved diagnosis of the plasma edge which will allow to test theoretical predictions. The second set of eight B-coils enables studies with $n=4$, i.e. stronger edge localization of the field and the possibility to rotate $n=2$ and $n=3$ modes toroidally, e.g. 


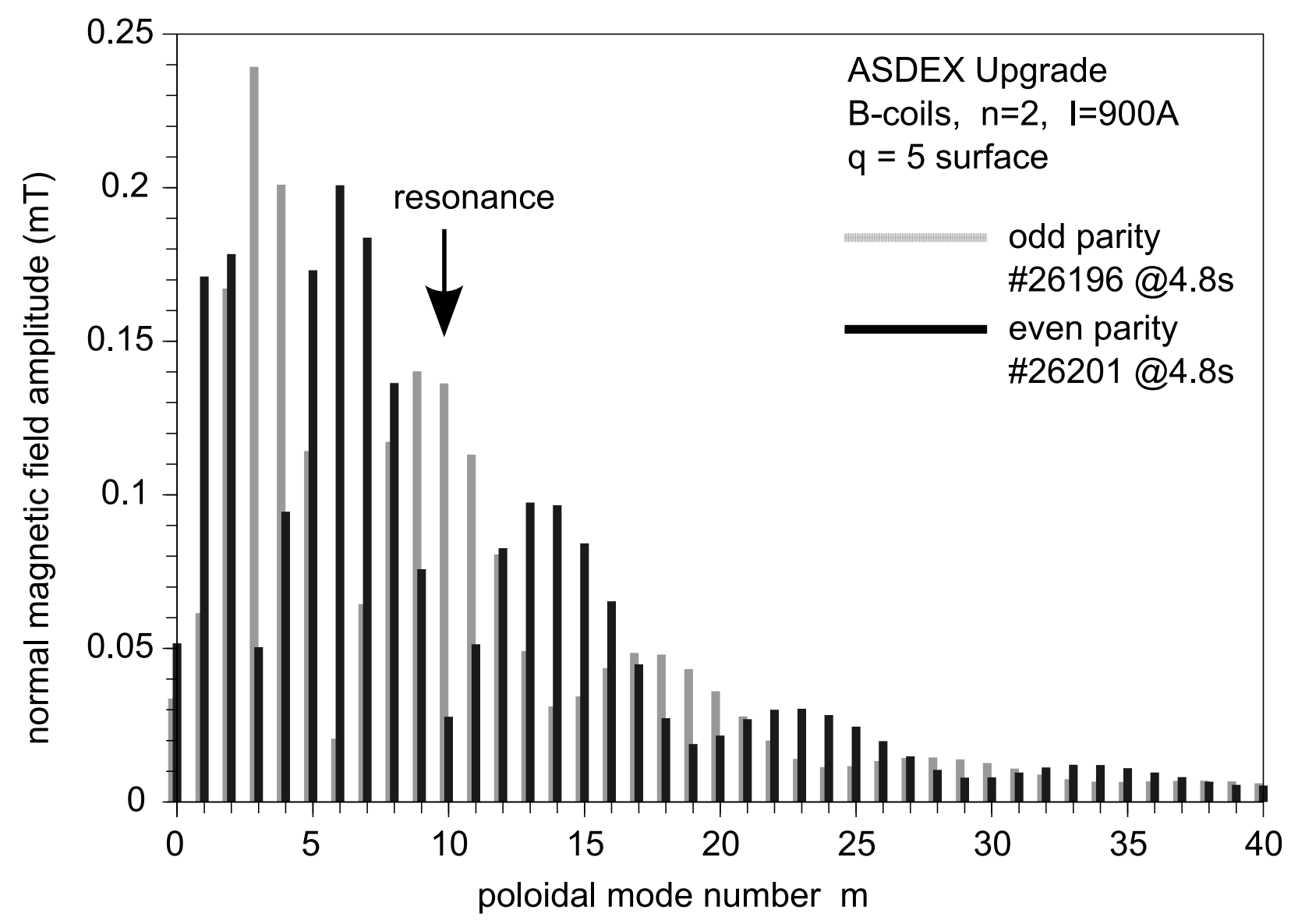

FIG. 7. Poloidal mode number $(m)$ spectra (taken for $n=2$ component at the $q=5$ surface) for discharges 26196 (odd parity) and 26201 (even parity).

for diagnostics purposes.

[1] G. Federici, Physica Scripta T124, 1 (2006)

[2] A. Loarte et al., Plasma Phys. Control. Fusion 45, 1549 (2003)

[3] T. C. Hender et al., Nucl. Fusion 32, 2091 (1992)

[4] Y. Liang et al., Phys. Rev. Lett. 98, 265004 (2007)

[5] T. E. Evans et al., Phys. Rev. Lett. 92, 235003 (2004)

[6] T. E. Evans et al., Nucl. Fusion 45, 595 (2005)

[7] W. Köppendörfer et al., in Plasma Physics and Controlled Nuclear Fusion Research 1992, Vol. 1 (IAEA, Vienna, 1993) pp. 127-140

[8] W. Suttrop et al., Fusion Eng. \& Design 84, 290 (2009) 
[9] T. Vierle et al., Fusion Eng. \& Design 84, 1928 (2009)

[10] M. Rott et al., Fusion Eng. \& Design 84, 1653 (2009)

[11] H. Zohm Plasma Phys. Control. Fusion 38, 105 (1996)

[12] A. Kallenbach et al., Plasma Physics and Controlled Fusion 52, 055002 (2010)

[13] T. Pütterich et al., Plasma Phys. Control. Fusion 50, 085016 (2008)

[14] M. Greenwald et al., Nucl. Fusion 28, 2199 (1988) 